\title{
Aprendizaje cooperativo y habilidades sociales: Universidad Nacional Jorge Basadre Grohmann
}

\section{Achap atipayninkuna yanapanakuna yaćhana: Universidad Nacional Jorge Basadre Grohmann}

\author{
Recepción: 09 julio 2020 Aprobación: 11 agosto 2020
}

Adriana Maximina Luque Ticona Nacionalidad: peruana / Universidad Nacional Jorge Basadre Grohmann

Correo: adrianaluquet@gmail.com

Isaías Rey Pérez Alférez Nacionalidad: peruano / Universidad Nacional Jorge Basadre Grohmann Correo: isaiasperez20o@gmail.com

Juan Antonio Aguilar Quispe Nacionalidad: peruano / Universidad Nacional Jorge Basadre Grohmann Correo:jaguilarq@unnjbg.edu.pe

María Ruth Rozas Flores Nacionalidad: peruana / Universidad Nacional Jorge Basadre Grohmann Correo:mariaruthrozas@gmail.com

\section{Resumen}

El presente trabajo se ejecutó en estudiantes de Educación y Ciencias de la Comunicación de la Universidad Nacional Jorge Basadre Grohmann-Tacna; con el propósito de conocer la relación entre el nivel del aprendizaje cooperativo con el desarrollo de habilidades sociales que permitan, aposteriori, visualizar el logro de aprendizajes significativos en los estudiantes. Se trabajó con una muestra de 93 estudiantes del 5 to año, distribuidos en 42 estudiantes de la Escuela Profesional de Ciencias de la Comunicación, y 51 pertenecientes a la Escuela Profesional de Educación. El diseño de investigación ha sido no experimental de tipo descriptivo correlacional. La verificación de las hipótesis de estudio, permitió aceptar y aprobar las hipótesis planteadas.

\section{Palabras clave:}

Aprendizaje cooperativo, habilidades sociales, estudiantes universitarios.

\section{Lisichiku limaykuna:}

Yanapanakuy yaćhakuna, achkap yaćhanankuna, hatunyaćhaywasip yaćhapakuninkuna 


\section{Cooperative Earning and Social Skills in Students of Education and Communication Sciences: National University Jorge Basadre Grohmann}

\begin{abstract}
The present work was carried out in students of Education and Communication Sciences of the Jorge Basadre Grohmann-Tacna National University; with the purpose of knowing the relationship between the level of cooperative learning with the development of social skills that allow, subsequently, to visualize the achievement of significant learning in students. We worked with a sample of 93 5th year students, distributed in 42 students of the Professional School of Communication Sciences, and 51 belonging to the Professional School of Education. The research design has been non-experimental of a descriptive correlational type. The verification of the study hypotheses allowed accepting and approving the hypotheses raised.
\end{abstract}

\section{Keywords}

Cooperative learning, social skills, college students.

\section{Aprendizagem cooperativa e habilidades sociais: Universidad Nacional Jorge Basadre Grohmann-Tacna}

\section{Resumo}

O presente trabalho foi realizado com estudantes das licenciaturas da Educação e de Ciências da Comunicação da Universidade Nacional Jorge Basadre Grohmann-Tacna; com o propósito de conhecer a relação entre o nível de aprendizagem cooperativa com o desenvolvimento de habilidades sociais que permitam, a posteriori, visualizar o alcançe de aprendizagens significativas nos estudantes. Trabalhou-se com uma amostra de 93 estudantes do $5^{\circ}$ ano, distribuídos em 42 estudantes da Escola Profissional de Ciências da Comunicação e 51 das Escolas Profissionais voltadas para a Educação. $O$ desenho da pesquisa foi do tipo descritivo-correlacional não experimental. A verificação das hipóteses do estudo permitiu aceitar e aprovar as hipóteses levantadas.

\author{
Palavras-chave: \\ Aprendizagem \\ cooperativa, \\ habilidades \\ sociais, estudantes \\ universitários.
}

\section{Datos de los autores}

Adriana Luque Ticona es docente principal e investigadora en educación, lengua y literatura y ciencias histórico-sociales. Doctora en Educación por la Universidad Católica Santa María de Arequipa. Magíster en Docencia Universitaria y Gestión Educativa por la Universidad Privada de Tacna.

Isaías Rey Pérez Alférez es docente universitario e investigador en educación, antropología y ciencias sociales. Antropólogo por la Universidad Nacional San Antonio Abad de Cusco. Magíster en Docencia Universitaria y Gestión Educativa por la Universidad Privada de Tacna.

Juan Antonio Aguilar Quispe, es decente universitario en matemáticas. Profesor de Estado por la Universidad de Chile. Magíster en Tecnología Educativa por la Universidad Nacional Jorge Basadre Grohmann.

María Ruth Rozas Flores, docente e investigadora en ciencias de la comunicación. Magister en Educación con mención en Currículum por la Universidad de Tarapacá (Arica, Chile) 


\section{Introducción}

En los tiempos actuales, la transformación global que vive la sociedad del siglo XXI, sobre todo los sistemas educativos vienen generando cambios frente a los retos de cómo asumir nuevas formas, no solo de incorporar habilidades de relación social, sino nuevas formas de aprendizaje.

En las universidades se observa gran número de estudiantes por aula, que en algunos casos dificultan la actividad académica. Además se advierte que la mayoría de los docentes, desarroIlan actividades bajo los modelos tradicionales que resultan siendo inadecuados, pasivos, una simple aplicación del saber transmitido; por lo que, frente a esta realidad, surge la necesidad que los docentes universitarios cuenten con herramientas pertinentes que les permita ir moldeando al futuro profesional e ir preparándolo para hacer frente a la complejidad de situaciones que diariamente se presentarán en su quehacer académico; por lo tanto, este aprendizaje debe contar con el asesoramiento del docente. Desde esta perspectiva el aprendizaje cooperativo es una herramienta valiosa para ir gestando cambios que deben propiciar los docentes, buscando establecer la correspondencia entre el aprendizaje cooperativo y las habilidades sociales, permitiendo hacer de su uso una constante en los espacios universitarios.

El aprendizaje cooperativo es importante, permite a los estudiantes desarrollar habilidades sociales. Bajo este contexto se hace necesario replantear los métodos y estrategias didácticas de enseñanza-aprendizaje, máxime si ahora el proceso sigue la línea del constructivismo pedagógico, donde el estudiante es el generador de su propio conocimiento, basado en las experiencias previas y el conflicto cognitivo en su proceso de construcción de los aprendizajes significados.

El presente trabajo pretende proporcionar un mayor conocimiento sobre el aprendizaje cooperativo como una alternativa en la formación de futuros profesionales comprometidos con su sociedad, solidarios, cooperantes, que establezcan un proceso comunicativo constante en la posibilidad de compartir conocimientos y solucionar problemas durante el proceso formativo profesional; además de proveer a los estudiantes la posibilidad de aprender por medio de la interacción con sus compañeros.

\section{El aprendizaje cooperativo}

Hablar del aprendizaje cooperativo -AC- es ingresar al tratamiento de un concepto diferente del proceso de enseñanza y aprendizaje habitual, arcaico y desfasado de hoy en día. Se basa en la interacción entre alumnos diversos, que en grupos de 4 a 6 , cooperan en el aprendizaje de distintas cuestiones de índole muy variada. Este aprendizaje cuenta con el asesoramiento de un maestro, que dirige este proceso supervisándolo. Se trata, pues, de un concepto del aprendizaje no competitivo ni individualista como es el método tradicional, sino un mecanismo colaborador que pretende desarrollar hábitos de trabajo en equipo, la solidaridad entre compañeros, y que los alumnos intervengan autónomamente en su proceso de aprendizaje (Benito, M y Mena, J.M., 2007).

Por su parte Johnson, D., Johnson, R. y Holubec, E. entienden la cooperación como el trabajar juntos para conseguir objetivos comunes. En este tipo de aprendizaje los estudiantes establecen una interdependencia positiva; es decir, solo podrán alcanzar sus objetivos si todos los miembros del grupo también lo hacen (1999, p. 5), 
Como se puede deducir, este tipo de metodología, contrasta las metodologías tradicionales de carácter competitivo e individualista; en éstas, los individuos también comparten objetivos y metas, pero crean una interdependencia negativa. Los estudiantes pueden alcanzar sus objetivos independientemente a sus compañeros los consigan o no (Torrego,J.A. y Negro, A (coordinadores), 2012. p. 16)

Otro de los autores contemporáneos más referenciados en nuestro contexto en términos de aprendizaje cooperativo es Pere Pujolàs Maset (2009) quien la define como el uso didáctico de equipos reducidos de alumnos, generalmente de composición heterogénea en rendimiento y capacidad, aunque ocasionalmente pueden ser homogéneos, aseguran al máximo la participación igualitaria (para que todos los miembros del equipo tengan una misma oportunidad de participar) y se potencia al máximo la interacción simultánea entre ellos.

Lo señalado es ratificado por Gemma Riera Romero, quien precisa que el aprendizaje cooperativo es un conjunto de procedimientos de enseñanza que parten de la organización de clase de pequeños grupos heterogéneos, a partir de los cuales los alumnos trabajan conjuntamente en forma coordinada para resolver tareas académicas y profundizar aprendizajes. Esta concepción enfatiza que los equipos deben ser pequeños y heterogéneos, y siempre garantizar la participación igualitaria o por lo menos equitativa de los integrantes, potenciando la interacción simultánea (2011).

Lo señalado por los autores precedentes, nos llevan a entender que el aprendizaje cooperativo descarga su efectividad sobre la forma en que el docente dinamiza los espacios de aprendizaje y propicia la profundización de la interacción entre estudiantes, lo cual podrá convertir una sesión de clase en una experiencia enriquecedora o en una aburrida actividad académica a la cual se asiste solo por obligación.

\section{Elementos esenciales del aprendizaje}

\section{cooperativo}

No todos los grupos que se forman para trabajar en clase se pueden llamar grupos cooperativos. Johnson, Johnson y Holubec (Johnson D. J., 1999. p. 6-7) establecieron cinco elementos fundamentales necesarios para que se produjera un verdadero aprendizaje cooperativo. Estos elementos también fueron descritos posteriormente por autores como Pujolás Maset (2009), y Torrego y Negro (2012):

a) La interdependencia positiva: Surge cuando los componentes del grupo se percatan que su trabajo está vinculado al de los demás y que solo podrán tener éxito si y solo si todos los componentes del grupo logran alcanzar sus objetivos propios. El objetivo final de la tarea no se podrá alcanzar si uno falla. Así todos necesitarán de la implicación de los demás.

b) Responsabilidad individual y grupal: Como ya hemos dicho, el éxito depende de todos los miembros del grupo, por eso es necesario un compromiso de grupo, donde todos asuman la responsabilidad de alcanzar los objetivos grupales, y otro individual donde cada uno se responsabilizará de sus objetivos propios. Este aspecto trata de evitar la difusión de responsabilidades. Nadie puede aprovecharse del trabajo de otros. 
c) Interacción estimuladora: Se trata de maximizar las posibilidades de interacción mediante dinámicas interpersonales. Los alumnos deben promover el éxito de los demás compartiendo, ayudando, respaldando y animando a sus compañeros. Los trabajos son producto del esfuerzo y aporte conjunta, basados en el compromiso y respeto por el otro.

d) Habilidades interpersonales y grupales: El aprendizaje cooperativo va más allá de aprender cualquier materia. Los alumnos deben adquirir destrezas interpersonales y de trabajo en grupo. Deberán comunicarse, tomar decisiones, resolver conflictos, organizarse y apoyarse entre ellos.

e) Evaluación grupal: Los alumnos se hacen responsables del proceso de evaluación. Destinarán un tiempo para reflexionar conjuntamente para evaluar el logro de sus objetivos y las relaciones de trabajo. Deberán tomar decisiones sobre aquello que necesitan reajustar o mejorar.

\section{Finalidad del aprendizaje cooperativo}

Según Cordero y Luna (2010), son finalidades que contempla el aprendizaje cooperativo:

a) La correlación positiva de logros;

b) La adquisición de objetivos compartidos;

c) El desarrollo de procesos de interacción;

d) La cooperación como elemento clave para el aprendizaje; y

e) La respuesta a la diversidad.

Esta clasificación del AC permite ubicar los diferentes enfoques por los que ha transitado su conceptualización, desde sus inicios hasta la actualidad: se parte de una situación cooperativa condicional, inherente a este proceso; es grupal, es decir, prima la organización del trabajo mediante grupos heterogéneos con objetivos compartidos; es relacionista, ya que un elemento fundamental es la relación interactiva que se produce en el equipo cooperativo; es motivacional, en tanto que parte de la visión del método como oportunidad para generar un impulso positivo hacia el aprendizaje; y es inclusivo, ya que se utiliza como un medio para responder a la diversidad del alumnado. El equipo de investigadores considera que el aprendizaje cooperativo actúa como una estrategia sistemática que pueden ser utilizadas en cualquier curso o nivel académico.

Ahora bien, debido a que la educación es fundamental para el desarrollo y progreso de individuos y sociedades, es evidente el interés por estudiar y comprender la educación con el fin de hacerla más eficiente y satisfactoria para así crear otras formas de instruir y dar a conocer el mundo. De ahí que el aprendizaje cooperativo surge como una alternativa que permite encontrar un nuevo espacio educativo y de enseñanza entre alumnos, gracias a que es posible concebir el aula como una comunidad de aprendizaje en la que las ayudas pedagógicas se proporcionan entre todos sus miembros (Duran D. y., 2006. p. 31). De la misma manera, se hace evidente que las situaciones de interacción, entre iguales, son un espacio ideal para que los alumnos utilicen plenamente las potencialidades del lenguaje como instrumento de aprendizaje, afianzándose en una forma diferente de enriquecer la actividad educativa, con la que es posible hacer una construcción conjunta y compartida de conocimiento, y fortalecer factores sociales, interpersonales, comunicativos y de carácter académico de manera que unos aprenden de otros potenciando habilidades que cada uno tiene. 
Lo señalado implica reconocer que el aprendizaje cooperativo es un modelo educativo y una metodología que ha sido utilizada como herramienta importante dentro de contextos educativos, pues su conjunto de principios, técnicas y estrategias, donde se enseña y se potencia el desarrollo de cada estudiante con la colaboración de los demás miembros de un equipo, no solo generando conocimientos o información, sino que también intensifica importantes habilidades de distintos tipos y naturaleza, así como las actitudes y valores (Ferreiro Gravié, 2004. p. 35).

Resumiendo, se puede afirmar que el aprendizaje cooperativo, que compromete a más de dos personas en el tratamiento de un tema, permite visualizar los lazos de solidaridad, reciprocidad, correspondencia, es decir el trato entre individuos que, a la postre, no son sino la manifestación de sus habilidades sociales.

\section{Habilidades sociales}

Al decir de Vicente E. Caballo, los seres humanos son sociables, ya que interactúan en diferentes ámbitos de sus vidas, desde la infancia hasta la adultez, en contextos familiares, amicales y profesionales. (...) siendo las habilidades sociales parte desde la vida diaria, es necesario llegar a conocerlas a propósito de cambiarlas y saber, en definitiva, cómo hacerlo (2007. p. 3-4).

En la actualidad existen diversos abordajes sobre el desarrollo de las habilidades sociales, como elemento clave para mejorar la calidad de las interacciones sociales en el campo educativo; sin embargo, existen algunas discordancias. Otero, López y Álvarez, afirman que se han dado numerosas definiciones sin llegar a un consenso y a conocer dos razones: a) dentro del concepto de habilidades sociales se incluyen varias conductas; b) las habilidades sociales dependen de un contexto social, el cual no es permanente, sino variable en esencia (2010. p. 3).

Jeffrey Kelly define a las habilidades sociales como un conjunto de conductas aprendidas, que emplean los individuos en las situaciones interpersonales para obtener o mantener el reforzamiento de su ambiente (2002: 19). Según este autor, esta definición presenta tres aspectos fundamentales: a) el hecho de que un comportamiento es socialmente hábil en la medida que implique consecuencias reforzantes del ambiente, b) tener en cuenta las situaciones interpersonales en las que se expresan las habilidades sociales y c) la posibilidad de describir las habilidades sociales de modo objetivo. De este modo, Kelly sostiene que las habilidades sociales son medios que tiene un sujeto para alcanzar sus objetivos; es decir, remarca la función que tienen las habilidades sociales para la resolución de situaciones interpersonales, lo que las torna necesarias para la adaptación al ambiente más próximo de la persona.

Si bien asumir una conceptualización unívoca o de mayor precisión es dificultosa sobre el tema, Vicente E. Caballo ofrece una definición que resulta comprensible al señalar que las habilidades sociales son un conjunto de conductas emitidas por un sujeto en un contexto interpersonal que expresa sentimientos, actitudes, deseos, opiniones o derechos de un modo adecuado a la situación, respetando esas conductas en los demás, y que generalmente resuelve los problemas inmediatos mientras minimiza la probabilidad de futuros problemas (2007. p. 3-4).

Tras estas concisas definiciones, se colige que las habilidades sociales son un componente importante en las relaciones que se establecen en el ámbito de estudiantes, de cualquier nivel o en el nivel de cualquier relación personal. La vida universitaria, implica el tratamiento de temas vinculantes, en función a la carrera profesional. El compartir conocimientos deviene, entonces, 
en una acción cotidiana. Sin embargo, ese compartir, generalmente no es canalizada de manera adecuada, es decir bajo una metodología que genere rentabilidad en el conocimiento. De allí que se hace necesario, la implementación de metodologías que posibiliten el accionar colectivo, participativo y de beneficio común para el estudiantado. Una forma de generar un buen provecho de estas habilidades es mediante la aplicación del aprendizaje cooperativo.

Cabe precisar que, en cuanto a habilidades sociales relevantes, se consideraron: la asertividad, comunicación, autoestima y toma de decisiones que tienen una estrecha relación con el desarrollo cognitivo y los aprendizajes de los estudiantes, además es requisito indispensable para una buena socialización. Las habilidades sociales no solo son importantes porque se refieren a las relaciones de estudiantes y docentes; sino que también permiten que los estudiantes asimilen los roles y normas sociales, logrando un aprendizaje cooperativo.

En el presente trabajo, precisamente se ha buscado, reflejar la realidad que se observa en la Facultad de Educación, Comunicación y Humanidades, máxime que la mayor muestra comprende a estudiantes de la carrera profesional de educación, es decir estudiantes que se forman para enseñar a estudiantes del nivel secundario, principalmente.

\section{Materiales y métodos}

El presente trabajo es una investigación que se inscribe en el enfoque cuantitativo, y éste dentro del tipo de investigación básica o pura, de naturaleza descriptiva correlacional, transeccional, no experimental, debido a que está basado en medir las propiedades de las variables en estudio: habilidades sociales y el aprendizaje cooperativo en los estudiantes de la Escuela Profesional de Educación (ESED) y Escuela Profesional de Ciencias de la Comunicación (ESCC) de la Facultad de Educación, Comunicación y Humanidades de la Universidad Nacional Jorge Basadre Grohmann.

Se ha tomado a la población total de estudiantes de la ESED y la ESCC asciende a 686. Considerando por escuelas, se tiene que la Escuela Profesional de Educación tiene 499 estudiantes y la Escuela Profesional de Ciencias de la Comunicación, un total de 187 estudiantes.

Para el presente trabajo, se ha optado por un muestreo intencional, por conveniencia, dado que son grupos ya constituidos. En ese sentido, se ha trabajado con 51 estudiantes del 5 to. año, de las especialidades de Ciencias Sociales y Promoción Socio-cultural, y Lengua y Literatura de la Escuela de Educación y 42 estudiantes de Comunicación, que tienen un mejor conocimiento del desempeño de sus docentes, según el siguiente detalle muestral:

Tabla 1

Especialidades

\begin{tabular}{|l|l|l|l|l|}
\hline \multirow{2}{*}{ Escuela Profesional } & \multicolumn{3}{|l|}{ Especialidades } & \multirow{2}{*}{ TOTAL } \\
\cline { 2 - 4 } & SPRO & LEGE & CC & \\
\hline Educación & 33 & 18 & & 51 \\
\hline $\begin{array}{l}\text { Ciencias de la } \\
\text { Comunicación }\end{array}$ & & & 42 & 42 \\
\hline TOTAL & 33 & 18 & 42 & 93 \\
\hline
\end{tabular}

FUENTE: Registros Académicos FECH 
Se aplicó la técnica de la encuesta, mediante el instrumento cuestionario, elaborado y validado (coeficiente alfa de Cronbach) de acuerdo a las normas investigativas generalmente aceptadas. Sobre el caso, se han elaborado dos cuestionarios: uno para la variable habilidades sociales, con cuatro dimensiones: asertividad, comunicación, autoestima y toma de decisiones, con un total de 42 indicadores; para la variable aprendizaje cooperativo se elaboró un cuestionario constituido por cinco dimensiones (habilidades sociales, procesamiento grupal, interdependencia positiva, interacción promotora y responsabilidad individual) con 20 indicadores.

Para el análisis estadístico, se utilizó la información recogida, procesada electrónicamente, mediante el programa Microsoft Excel versión XP bajo ambiente Windows, SPSS (versión 20), que permitió la presentación de los Ítems mediante tablas y gráficas y, al mismo tiempo estimar las frecuencias absolutas y relativas de cada reactivo para describir las variables en estudio. Una vez procesada la información, se procedió a la verificación de las hipótesis. Para la contrastación se empleó el programa estadístico del chi cuadrado.

\section{Resultados}

La información procesada (presentadas en tablas del 1 al 18 y resumidas en las tablas 19 y 20) arrojan los siguientes resultados, por dimensiones:

- El 38,1\% de los estudiantes de la ESCC, posee un nivel alto en la dimensión de asertividad de la variable habilidades sociales, el 35,7 \% regular y el 26,2 \% nivel bajo. En la ESED, el 35,3\% posee un nivel bajo, el 33,3\% un nivel regular y el 31,4 \% posee un nivel alto.

- El 42,9\% de los estudiantes de la ESCC, posee un nivel alto en la dimensión comunicación de la variable habilidades sociales, el 38,1 \% nivel regular y el 19,0 \% nivel bajo. En la ESED el 45,1 $\%$ posee un nivel regular, el 33,3 \% se ubica en un nivel alto y el 21,6 \% posee un nivel bajo.

- El 42,9\% de los estudiantes de la ESCC, posee un nivel bajo en la dimensión de autoestima de la variable de habilidades sociales, el 35,7\% nivel regular y el 21,4 \% nivel alto. En la ESED el $37,3 \%$ posee un nivel bajo, el $33,3 \%$ posee un nivel regular y el $29,4 \%$ posee un nivel alto.

- El 35,7 \% de los estudiantes de la ESCC, posee un nivel alto en la dimensión de toma de decisiones de la variable habilidades sociales, el 33,3\% un nivel bajo y el 31,0 \% nivel regular. En La ESED, el 41,2 \% posee un nivel bajo, el 33,3\% un nivel regular y el $25,5 \%$ un nivel alto.

- El 50,0\% de los estudiantes de la ESCC, posee un nivel regular en la dimensión de habilidades sociales, mientras que el $26,2 \%$ posee un nivel bajo y el $23,8 \%$ posee un nivel alto. En la ESED, el $37,2 \%$ se ubica en un nivel bajo, el 31,4 \% en nivel alto y el 31,4 \% en el un nivel regular.

- El 42,9 \% de los estudiantes de la ESCC, posee un nivel bajo en la dimensión de procesamiento grupal de la variable aprendizaje cooperativo, el 38,1 \% un nivel regular y el 19,0 \% un nivel alto. En la ESED el $37,3 \%$ posee un nivel regular, el $33,3 \%$ un nivel bajo y el $29,4 \%$ un nivel alto.

- El 42,9\% de los estudiantes de la ESCC, muestra un nivel alto en la dimensión interdependencia positiva, el $36,6 \%$ un nivel regular y el $20,7 \%$ un nivel bajo. En la ESED, el 39,2 \% se ubica en un nivel regular, el 31,4 \% posee un nivel bajo el $29,4 \%$ posee un nivel alto.

- El 42,9\% de estudiantes de la ESCC, posee un nivel regular en la dimensión interacción promotora de la variable aprendizaje cooperativo, el 33,3 \% y el 23,8 \% posee un nivel alto. En la ESED, el $35,3 \%$ posee un nivel bajo, el $33,3 \%$ un nivel regular y el $31,4 \%$ posee un nivel alto.

- El 40,4 \% de estudiantes de la ESCC, muestran un nivel regular en la dimensión responsabilidad individual de la variable aprendizaje cooperativo, el 31,0 \% un nivel alto y el $28,6 \%$ un nivel bajo. En la ESED, el 35,3 \% posee un nivel alto, el 33,3\% un nivel regular y el 31,4 \% un nivel bajo. 
Los resultados anteriores, de manera consolidada, para las dos muestras (ESED y ESCC), representadas en niveles, indican el siguiente resultado:

\section{Tabla 2}

Resultado de aplicar el cuestionario para determinar el nivel de HABILIDADES SOCIALES de los estudiantes de la ESED y ESCC.

\begin{tabular}{lll}
\hline Nivel & Frecuencia & Porcentaje \\
\hline Nivel Bajo & 28 & $30,1 \%$ \\
\hline Nivel Medio & 35 & $37,6 \%$ \\
Nivel Alto & 30 & $32,3 \%$ \\
\hline TOTAL & 93 & $100.0 \%$ \\
\hline
\end{tabular}

FUENTE: Cuestionario aplicado a los estudiantes de la muestra

\section{Gráfica 1}

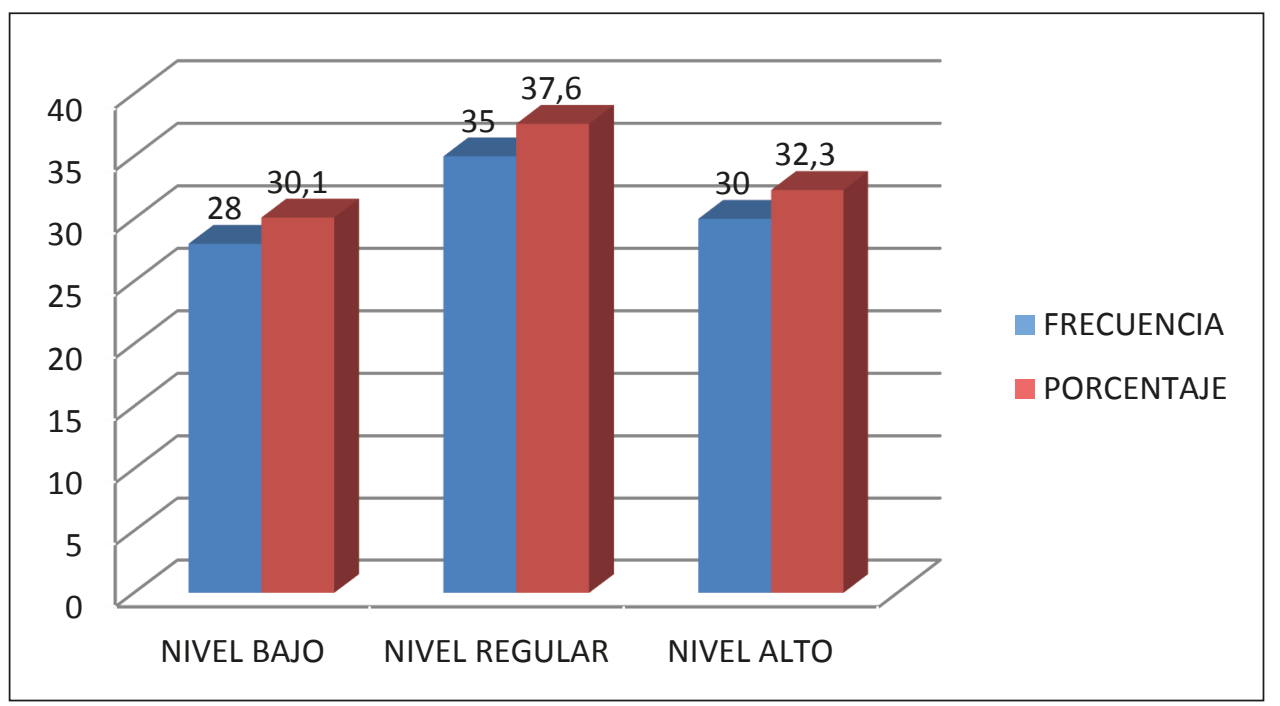

La información procesada indica que los estudiantes de la muestra, en un 37,6\% consideran que el nivel de habilidades sociales es regular; el 30,1\% es bajo, y el 32.3\% tienen un nivel alto.

\section{Tabla 3}

Resultado de aplicar el cuestionario para determinar el nivel de aprendizaje cooperativo de los estudiantes de la ESED y ESCC.

\begin{tabular}{lll}
\hline NIVEL & FRECUENCIA & PORCENTAJE \\
\hline Nivel Bajo & 27 & $29,0 \%$ \\
\hline Nivel Medio & 35 & $37,7 \%$ \\
\hline Nivel Alto & 31 & $33,3 \%$ \\
\hline TOTAL & 93 & $100.0 \%$ \\
\hline
\end{tabular}

FUENTE: Cuestionario aplicado a los estudiantes de la muestra 


\section{Gráfica 1}

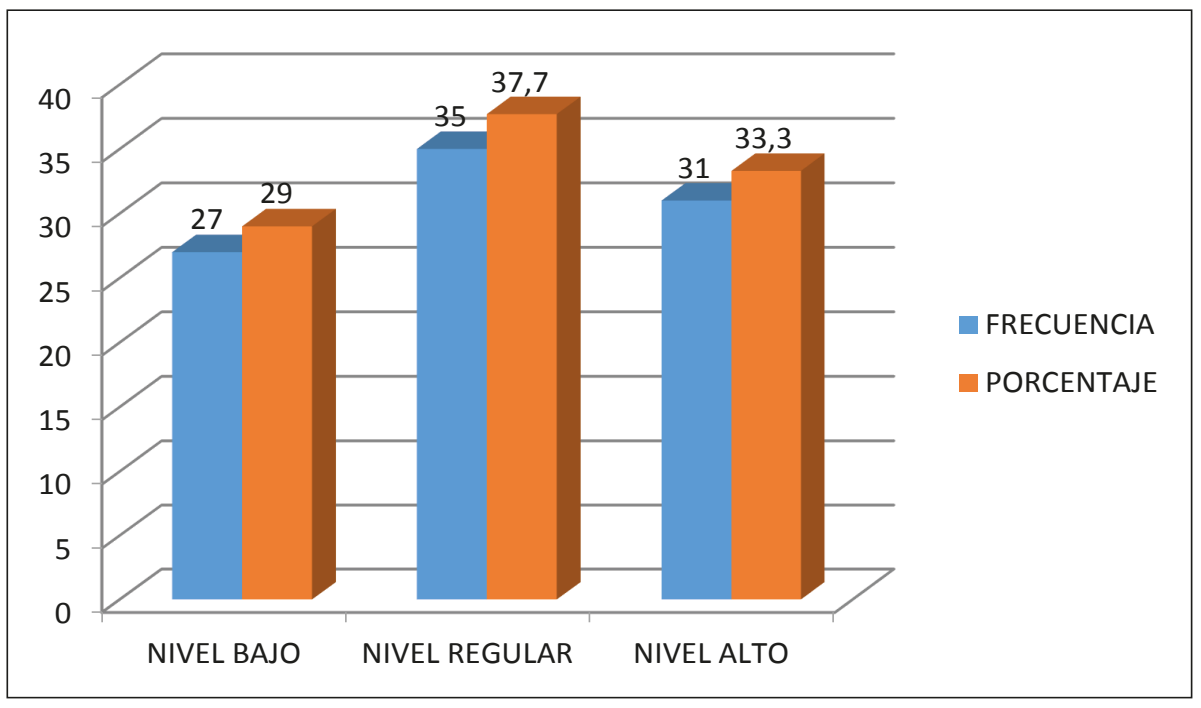

Los resultados indican que el $37,7 \%$ de los estudiantes se ubican en un nivel regular de aprendizaje cooperativo, el 33,3 en el nivel alto, y, el 29,0 5 en el nivel bajo.

\section{Tabla de contingencia}

Para analizar la relación de dependencia o independencia entre dos variables cualitativas nominales o factores, es necesario estudiar su distribución conjunta o tabla de contingencia.

Nuestras variables son las habilidades sociales y el aprendizaje cooperativo, en los estudiantes de la ESCC y ESED.

Tabla 4

\begin{tabular}{lllll}
\hline NIVEL & & & & \\
& BAJO & MEDIO & ALTO & \\
ESCC & 11 & 12 & 19 & $\mathbf{4 2}$ \\
ESED & 15 & 26 & 10 & $\mathbf{5 1}$ \\
& 26 & 38 & $\mathbf{2 9}$ & $\mathbf{9 3}$ \\
\hline
\end{tabular}

Estas tablas tienen dos objetivos fundamentales:

1. Organiza la información de la investigación, cuando está referida a dos variables cualitativas.

2. A partir de la tabla de contingencia se puede analizar si existe una relación de dependencia o independencia entre las dos variables cualitativas sujetas de estudio.

Como se puede observar, el cruzamiento de los datos, sobre los resultados de ambas variables, confirman que el nivel de habilidades sociales y el nivel de aprendizaje cooperativo, guardan una relación directa, lo que permite confirmar lo planteado por la hipótesis de estudio, tal como se corrobora a continuación con la contrastación respectiva. 


\section{Verificación de las hipótesis}

\section{Hipótesis específica "a”}

"El nivel de habilidades sociales que evidencian los estudiantes de la ESED y ESCC es regular"

Para llegar a comprobar la hipótesis específica “a”, se trabajó con la tabla 2.

\section{Tabla 5}

\begin{tabular}{lll}
\hline Nivel & Frecuencia & Porcentaje \\
\hline Nivel Bajo & 28 & $30,1 \%$ \\
\hline Nivel Medio & 35 & $37,6 \%$ \\
\hline Nivel Alto & 30 & $32,3 \%$ \\
\hline TOTAL & 93 & $100.0 \%$ \\
\hline
\end{tabular}

Los estudiantes de la ESED y ESCC evidencian un nivel regular de habilidades sociales.

\section{Hipótesis específica "b"}

"El nivel de aprendizaje cooperativo que evidencian los estudiantes de la ESED y ESCC es regular"

Para llegar a comprobar la hipótesis específica “b”, se trabajó con la tabla 3.

Tabla 6

\begin{tabular}{lll}
\hline NIVEL & FRECUENCIA & PORCENTAJE \\
\hline Nivel Bajo & 27 & $29,0 \%$ \\
\hline Nivel Medio & 35 & $37,7 \%$ \\
\hline Nivel Alto & 31 & $33,3 \%$ \\
\hline TOTAL & 93 & $100.0 \%$ \\
\hline
\end{tabular}

De la lectura de los resultados se desprende que los estudiantes de la ESCC y de la ESED alcanzan un nivel medio en cuanto a sus habilidades de aprendizaje cooperativo.

\section{Hipótesis específica "c"}

"A regular nivel de habilidades sociales, regular nivel de aprendizaje cooperativo en los estudiantes de la ESED y ESCC, año 2018".

Como tenemos dos variables categóricas veremos su correlación utilizando el estadístico chi cuadrado, tenemos entonces dos hipótesis:

Hipótesis nula

$\mathrm{H}_{\mathrm{o}}$ : No existe la relación a regular nivel de habilidades sociales, regular nivel de aprendizaje cooperativo en los estudiantes de la ESED y ESCC año 2018.

Hipótesis alternativa

$\mathrm{H}_{\mathrm{a}}$ : Existe la relación a regular nivel de habilidades sociales, regular nivel de aprendizaje cooperativo en los estudiantes de la ESED y ESCC año 2018. 
Recordemos la tabla de contingencia (de doble entrada)

Tabla 7

De contingencia

\begin{tabular}{lllll}
\hline NIVEL & \multicolumn{1}{l}{ MEDIO } & ALTO & \\
& BAJO & 12 & 19 & $\mathbf{4 2}$ \\
ESCC & 11 & 26 & 10 & $\mathbf{5 1}$ \\
ESED & 15 & $\mathbf{3 8}$ & $\mathbf{2 9}$ & $\mathbf{9 3}$ \\
& $\mathbf{2 6}$ & & \\
\hline
\end{tabular}

Esta tabla de dos entradas, de las variables categóricas, nos muestra los valores obteni$\operatorname{dos} \mathrm{O}_{\mathrm{ij}}$

Los grados de libertad del chi cuadrado están dadas por $(3-1)(2-1)=2$

Luego $X_{0 ; 95,2)=5,9915}^{2}$

Recordemos ahora que los valores esperados se obtienen por la fórmula:

$e_{i j=}$ (total fila i)(total columna j)/380, lo que nos sirve para calcular el valor de

$X_{c a l}^{2}=\sum_{i} \sum_{j} \frac{\left(o_{i j}-e_{i j}\right)^{2}}{e_{i j}}$

Lo cual nos origina la siguiente matriz de valores esperados:

Tabla 8

Tabla de valores esperados

\begin{tabular}{|l|l|l|l|}
\hline NIVEL & MEDIO & ALTO \\
\hline & BAJO & 17.1612903 & 13.0967742 \\
\hline ESCC & 11.7419355 & 20.8387097 & 15.9032258 \\
\hline ESED & 14.2580645 & \\
\hline
\end{tabular}

FUENTE: Tabla 3 y fórmula dada

Donde $X_{\text {cal }}^{2}=7.76816583$

Y como 7.76816583 es mayor que 5,9915 se toma la hipótesis alternativa con lo que se prueba la tercera hipótesis específica:

Existe correlación entre la aplicación de las estrategias didácticas, por parte de los docentes en el proceso de enseñanza-aprendizaje y el nivel de aprendizaje significativo en los alumnos del cuarto y quinto año de la Escuela Profesional de Educación.

La hipótesis alternativa incluye a la hipótesis específica:

A menor nivel de aplicación las estrategias didácticas de los docentes en el proceso enseñanza-aprendizaje, menor nivel de aprendizaje significativo de los estudiantes del 5 to. año de las escuelas profesionales de la muestra. 
Hipótesis general:

"Las habilidades sociales se relacionan en forma directa con el aprendizaje cooperativo en los estudiantes de la ESED y ESCC, año 2018".

Es decir, al haberse aceptado y comprobado las tres hipótesis específicas, la hipótesis general queda plenamente confirmada. Con ello se corrobora el supuesto que ha generado la presente investigación.

\section{Discusión}

En la Tabla 2 se presenta la información procesada y consolidada de los resultados sobre la variable habilidades sociales de las dos escuelas. Los resultados indican que un $37,6 \%$ consideran que el nivel de habilidades sociales es regular. Ello confirma y acepta la primera hipótesis planteada. Sin embargo, este resultado contrasta con aquellas apreciaciones planteadas por estudiosos sobre la materia. Así, para Vicente, E. Caballo (2007) las habilidades sociales son un conjunto de conductas que permiten al individuo desarrollarse en un contexto individual o interpersonal expresando sentimientos, actitudes, deseos, opiniones o derechos de un modo adecuado a la situación. Generalmente, posibilitan la resolución de problemas inmediatos y la disminución de problemas futuros en la medida que el individuo respeta las conductas de los otros.

Holst Morales (2017), manifiesta que Daniel Goleman, Ingrid Cecilia Holst Morales, Yadira Galicia Barrera, Gabriela Gómez Veytia y Arely Degante González, plantean que la importancia de las habilidades sociales o de las relaciones interpersonales radica en que permite obtener éxito en el desempeño profesional y de vida; debido a que como seres sociales, las personas están en constante interacción con su entorno familiar, educativo y laboral por lo que es necesario que se adapten a ellos sin interferir en las relaciones que tienen los demás con sus propios entornos. De esta manera, las habilidades sociales son consideradas como destrezas para una adecuada y pertinente actuación social, que se logran a partir de un intercambio cultural y de conocimientos dentro de la vida cotidiana.

Lo señalado por los autores mencionados coinciden en la importancia que debe o se atribuye a las habilidades sociales, en todo nivel, en este caso más precisamente en el campo universitario. Obviamente que los fundamentos argüidos no coinciden plenamente con los resultados alcanzados en el presente trabajo. Se comprobó que el nivel de desarrollo y aplicación de las habilidades sociales en la Escuela Profesional de Educación (ESED) y Escuela Profesional de Ciencias de la Comunicación (ESCC) eran regulares. Los resultados comparados con la literatura exigen la asunción de nuevas ópticas en el manejo de la interrelación en aula, de manera que los estudiantes, mejor socializados, puedan asumir responsabilidades que los comprometa a un manejo de relaciones eficaces y eficientes y que les permita un desarrollo interpersonal y social adecuadas, así como elevar el nivel de aprendizaje durante su proceso de formación profesional.

En lo que se refiere a la segunda hipótesis específica: El nivel de aprendizaje cooperativo que presentan los estudiantes de la Escuela Profesional de Educación (ESED) y Escuela Profesional de Ciencias de la Comunicación (ESCC), es regular, la información procesada demuestra que su formulación corresponde con la realidad. En la Tabla 3, se confirma que el nivel de aprendizaje cooperativo de los estudiantes de las ESCC y ESED mayoritariamente corresponden a un $37,7 \%$, es decir se ubican en un nivel medio o regular. 
Thania M. y Oberto M. (2014), reconocen las dificultada des que pueden presentarse en el aula, pero precisan que "En las aulas universitarias es común ver secciones cada vez más numerosas, donde un docente debe realizar su labor educativa con una diversidad de estudiantes, lo cual en muchos casos dificulta el alcance básico de los objetivos instruccionales e impide el debido abordaje de los contenidos programáticos establecidos". Estos problemas pueden ser minimizados si el docente aplica estrategias de aprendizaje cooperativo que le permitan ese cambio de conducta esperado y generen en los estudiantes no solamente nuevas aptitudes, sino también actitudes positivas. Consideran que el aprendizaje cooperativo ofrece múltiples ventajas al docente, por cuanto el intercambio de información entre los propios estudiantes con un lenguaje que le es común, puede facilitar la comprensión de contenidos complejos, aunado al apoyo que los alumnos más aventajados pueden proporcionar al resto de los integrantes del equipo, incrementando la motivación hacía la obtención del conocimiento, mejorando así el rendimiento académico, contribuyendo a establecer relaciones entre los estudiantes que permitan un clima grupal armonioso y logrando los objetivos instruccionales de la clase.

Por su parte Gemma Riera Romero (2019), considera que el trabajo cooperativo y el aumento de la interacción entre el alumnado y entre el profesorado ha sido considerado desde siempre una clave educativa para la renovación pedagógica. Asimismo, explica que la sociedad tiene la necesidad de que todas las personas aprendan de manera cooperativa para hacer frente al individualismo que es perjudicial para las relaciones tanto sociales como escolares, ya que genera la competitividad y da lugar a que sólo unos cuantos alcancen el éxito. Bajo esos conceptos, Gemma Riera, adelanta que, si entendemos la cooperación como un proceso social de construcción del conocimiento, consideramos el aprendizaje cooperativo como un proceso en el que cada individuo aprende más de lo que aprendería por sí mismo, porque desde esta perspectiva el aprendizaje se construye en la interacción con otros individuos de un grupo de iguales.

Lo señalado, tanto por Thania y Oberto, así como por Rierra Romero, resalta la estrategia del aprendizaje cooperativo como una alternativa importante en el proceso formativo del estudiante. Los resultados encontrados en el presente trabajo, obviamente, no van por ese camino. Existen limitaciones respecto a cómo debe ser formado el estudiante, respecto a las habilidades sociales y el aprendizaje cooperativo propiamente dicho.

Evidentemente que ello implica aceptar que el desarrollo de estas competencias es aún limitado. Sin embargo, queda la responsabilidad de trabajar para su aplicación y desarrollo: los estudiantes serán los beneficiados.

Referente a la tercera hipótesis específica, según la información procesada que se presenta:

Tabla 9

Tabla de valores esperados

\begin{tabular}{|l|l|l|l|}
\hline \multicolumn{5}{|l|}{ NIVEL } & MEDIO & ALTO \\
\hline & BAJO & 17.1612903 & 13.0967742 \\
\hline ESCC & 11.7419355 & 20.8387097 & 15.9032258 \\
\hline ESED & 14.2580645 &
\end{tabular}

Donde $X_{\text {cal }}^{2}=7.76816583$

Y como 7.76816583 es mayor que 5,9915 se toma la hipótesis alternativa con lo que se prueba la tercera hipótesis específica: Existe correlación entre la aplicación de las estrategias di- 
dácticas, por parte de los docentes en el proceso de enseñanza-aprendizaje y el nivel de aprendizaje significativo en los alumnos del quinto año de la Escuela Profesional de Educación.

Si analizamos nuestras actividades diarias, podemos comprobar que una gran parte del tiempo lo pasamos relacionándonos con otras personas. Así, el grado de habilidad social que poseamos determinará la calidad de las relaciones que mantengamos con nuestra familia, con nuestros amigos, con nuestra pareja, con las personas que se encuentran en los lugares donde trabajamos y con aquellas con las que nos relacionamos en los distintos servicios de la comunidad. Se ha demostrado que, en concreto, la competencia social en la infancia está estrechamente relacionada con la adaptación social, académica y psicológica, tanto en edades infantiles como posteriormente en la edad adulta. La incompetencia social en la infancia, sin embargo, se relaciona con una baja aceptación o rechazo de los iguales y un aislamiento social, bajos niveles de rendimiento académico, inadaptación escolar, baja autoestima, desajustes psicológicos y problemas de salud mental en la adolescencia y en la edad adulta, entre otros.

Finalmente, se planteó como hipótesis general que "Las habilidades sociales de los docentes se relacionan en forma directa con el nivel de aprendizaje cooperativo en los estudiantes de la ESED y ESCC, año 2018", hecho que se ha aceptado y aprobado mediante los resultados analizados de las hipótesis específicas; por tanto, las hipótesis formuladas inicialmente han sido plenamente validadas, quedando como resultados en tanto las habilidades sociales como el aprendizaje cooperativo en los estudiantes del Quinto Año de las escuelas profesionales de Ciencias de la Comunicación y de Educación, presentan un nivel medio o regular, lo que implica una revisión de las estrategias didácticas para el mejoramiento de la formación del futuro profesional.

Los resultados alcanzados tras el análisis de los datos proporcionados por los estudiantes de la muestra, confirman los supuestos de los que parte en este trabajo de investigación. Estos supuestos permitieron establecer la existencia de una relación directa entre las habilidades sociales con el aprendizaje cooperativo.

\section{Conclusiones}

Se ha comprobado y aceptado que el nivel de habilidades sociales que evidencian los estudiantes de la ESED y ESCC es regular. La información procesada indica que los estudiantes de la muestra, en un 37,6 \% consideran que el nivel de habilidades sociales es regular, el $30,1 \%$ es bajo, y, el 32,3 \% tienen un nivel alto, de habilidades sociales (Tabla 19).

Se ha comprobado y aceptado que el nivel de aprendizaje cooperativo que presentan los estudiantes de la ESED y ESCC, es regular. En cuanto al nivel del aprendizaje cooperativo, los estudiantes en un $37,7 \%$ indican que tienen un nivel medio en el uso del aprendizaje cooperativo; el 29,0\% se califican como nivel bajo; y, el 33,3\% se califican con un nivel alto en el aprendizaje cooperativo (Tabla 20).

Se ha comprobado y aceptado que, a regular nivel de habilidades sociales, regular nivel de aprendizaje cooperativo en los estudiantes de la ESED y ESCC. Los datos presentados en la tabla de contingencia permiten verificar que el comportamiento de las variables se relaciona en forma directa con lo que, se confirma y acepta la hipótesis específica.

Los resultados precisan que las habilidades sociales se relacionan en forma directa con el aprendizaje cooperativo que se observa en los estudiantes de las escuelas profesionales de Educación y Ciencias de la Comunicación.

Agradecimiento. A la Universidad Nacional Jorge Basadre Grohmann-Tacna que, mediante el Vicerrectorado de Investigación, brindó apoyo a la ejecución del presente trabajo. 


\section{Referencias bibliográficas}

Benito, M y Mena, J.M. (2007). La educación Pública Prioritaria en la Comunidad de Madrid. Recuperado el 23 de noviembre de 2018 , de https://ticyaprendizajecooperativo.wikispaces. com/.../Aprendizaje\%2oCooperativo.pps

Caballo, M. (2007. p. 3-4). Manual de evaluación y entrenamiento de habilidades sociales. Madrid: Siglo $\mathrm{XXI}$.

Caballo, V. (2007). Manual de evaluación y entrenamiento de las habilidades sociales. S+etima edición. España: Siglo XXI.

Cordero, G. y. (2010). Encuesta retos de la evaluación de la evaluación de los programas de formación de profesores: el caso de un programa en metodps de aprenmdizaje cooperativo. Revista Iberoamericana de Evaluación Educativa 2010 - Volumen 3, Número 1e, 192-201.

Duran, D. y. (2003). Cooperar para enseñar y aprender. Cuadernos de Pedagogía No. 331, 74-76. Obtenido de https://core.ac.uk/download/pdf/78543899.pdf

Duran, D. y. (2006. p. 31). Tutoría entre iguales: de la teoría a la práctica. Un método de aprendizaje cooperativo para la diversidad en secundaria. Barcelona: Graó.

Ferreiro Gravié, R. (2004. p. 35). Estrategias Didácticas del Aprendizaje Cooperativo. México: Trillas.

Holst Morales, I. y. (2017). Las habilidades sociales y sus diferencias en estudiantes universitarios. VERTIENTES Revista Especializada en Ciencias de la Salud, 22-29,.

Jhonson y Jhonson, D. (1999). El aprendizaje cooperativo en el aula. Buenos Aires: Paidós.

Johnson, D. J. (1999. p. 6-7). El aprendizaje cooperativo en el Aula. Buenos Aires: Paidós.

Johnson, D., \& Jhonson, T. y. (1999, p. 5). El aprendizaje cooperativo en el aula. Buenos Aires: Paidós.

Johnson, D., \& Johnson, R. y. (1997). El Aprendizaje Cooperativo regresa a la Universidad: ¿qué evidencia existe de que funciona? Minesota: Universidad de Minesota.

Kelly, J. (2002: 19). Entrenamiento de las habilidades sociales. Bilbao: Dessclée de Brouwer.

Otero, M., \& López, S. y. (2010. p. 3). Ciencias Psicosociales. Santander, España: Universidad de Cantabria. Obtenido de Recuperado de http://ocn.unicam.es/ciencias-de-la-salud/cienciapsicosociales-i

Pujolás, P. (2009). La calidad en los equipos de aprendizaje cooperativo.Algunas consideraciones parfa el cálculo del grado de cooperatividad. Revista de Educación No. 349, 225-239. Recuperado el 12 de enero de 2019, de http://www.revistaeducacion.educacion.es/re349/re349_11.pdf

Pujolás, P. (2009). La calidad en los equipos de aprendizaje cooperativo.Algunas consideraciones parfa el cálculo del grado de cooperatividad. Revista de Educación No. 349, 225-239.

Riera Romero, G. (2011). El aprendizaje cooperativo como metodología clave para dar respuesta a la diversidad del alumnado desde un enfoque inclusivo. Revista Latinoamericana de Educación Inclusiva, 5(2), 133-149. Recuperado el 30 de setiembre de 2018, de http://www.rinace.net/rlei/ numeros/vol5-num2/art7

Riera Romero, G. (18 de enero de 2019). El aprendizaje cooperativo como metodología clave para dar respuesta a la diversidad del alumnado desde un enfoque inclusivo. Obtenido de http:// conexiones.dgire.unam.mx/wp-content/uploads/2017/og/Aprendizaje-cooperativo-comometodologia.pdf

Thania, M. y. (2014). El aprendizaje cooperativo como herrqamientapara la educación universitaria. Revista de Educación en Valores, Vol 1, No. 21, 50-69. Recuperado el 12 de enero de 2018, de http://servicio.bc.uc.edu.ve/multidisciplinarias/educacion-en-valores/vin21/artog.pdf

Torrego,J.A. y Negro, A (coordinadores). (2012. p. 16). Aprendizaje cooperativo en las aulas: Fundamentos y recursos para su implementación. Madrid: España: Alianza editorial. 